\title{
ENTIRE FUNCTIONS WITH SPIRAL LIMITS
}

\author{
R. B. BURCKEL and SADAHIRO SAEKI
}

It is (perhaps) well-known that given $0=t_{0}<t_{1}<\ldots<t_{n}=2 \pi$ and complex numbers $c_{1}, \ldots, c_{n}$, there exists an entire function $f$ such that $\lim _{r \rightarrow \infty} f\left(r e^{i t}\right)=c_{j}$ for each $t \in\left(t_{j-1}, t_{j}\right)$ and each $j=1,2, \ldots, n$. (See G. Pólya [7] and Exercises IV 185-IV 186 of G. Pólya and G. Szegó [8].) In a similar vein K. Grandjot [5] proved the existence of a non-zero entire function $f$ such that $f(z) \rightarrow 0$ as $z \rightarrow \infty$ along any algebraic curve (cf. also H. Bohr [2]). The review Mathematical Reviews $52 \# 8433$ gives a brief historical account of this subject; it should be supplemented by Paragraphs 21 and 43 of the encyclopedia article [1] of L. Bieberbach. For some related results, we mention the anecdotal paper [10] by W. J. Schneider. In this note, we shall construct an entire function with "spiral" limits, where the limits are finitely many preassigned polynomials. Our method uses the well-known technique of shoving poles to infinity.

For each $p>0$, let $S_{p}$ denote the class of all continuously differentiable realvalued functions $\theta$ on $(0, \infty)$ such that

$$
\int_{1}^{\infty}\left|\theta^{\prime}(t)\right| \cdot t^{-p} d t<\infty
$$

Thus every function of the form $P(t) \cos Q(t)+R(t) \sin S(t)$ belongs to $S_{p}$ for some $p>1$, where $P, Q, R, S$ are polynomials with real coefficients. By a spiral region we mean an open set in the complex plane $C$ of the form

$$
\Omega=\left\{r e^{i t}: r>0 \text { and } \theta_{1}(r)<t<\theta_{2}(r)\right\},
$$

where $\theta_{1}, \theta_{2} \in S_{p}$ for some $p>0$ and $\theta_{1}(t)<\theta_{2}(t) \leqq \theta_{1}(t)+2 \pi$ for all $t>0$. As is customary, we shall often identify a curve with its image set.

Theorem. Let $\Omega_{1}, \ldots, \Omega_{k}$ be pairwise disjoint spiral regions, $E_{j} \subset \Omega_{j}$ unbounded closed subsets of $C, P_{j}(z)$ polynomials in $z \in C$ for $j=1,2, \ldots, k$, and $N$ a natural number. Then there exists an entire function $g$ such that

$$
g(z)=P_{j}(z)+o\left(|z|^{-N}\right) \text { as } z \in E_{j} \text { tends to } \infty
$$

for each $j=1,2, \ldots, k$.

Corollary. Let $P$ be a polynomial, and $N$ a natural number. Then there exists a non-polynomial entire function $h$ such that $h(z)=P(z)+o\left(|z|^{-N}\right)$ as $z \rightarrow \infty$ along any algebraic curve.

doi:10.5186/aasfm.1984.0904 
To prove these results, we need three lemmas.

Lemma 1. Suppose that $\theta_{1}, \theta_{2} \in S_{p}$ for some $p>1$, and that $\theta_{1}<\theta_{2}<\theta_{3}$ on $(0, \infty)$, where $\theta_{3}=\theta_{1}+2 \pi$. For $j=1,2$, let

$$
\Omega_{j}=\left\{r e^{i t}: r>0 \text { and } \theta_{j}(r)<t<\theta_{j+1}(r)\right\},
$$

and let $g_{j}$ be a holomorphic function on $\Omega_{j}$ such that

$$
z \in \Omega_{j} \text { and }|z|>C \Rightarrow\left|g_{j}^{\prime}(z)\right|<C /|z|^{2 N}
$$

for some $C$ and $N>p+1$. Then there exist $c_{j} \in C$ such that

$$
g_{j}(z)=c_{j}+o\left(|z|^{-N}\right) \quad \text { as } \quad z \in \Omega_{j} \quad \text { tends to } \quad \infty(j=1,2) .
$$

Proof. For $j=1,2$ and $t \geqq 0$, define

$$
\tau_{j}(t)=2^{-1}\left\{\theta_{j}(t)+\theta_{j+1}(t)\right\} \text { and } \gamma_{j}(t)=t e^{i \tau_{j}(t)} .
$$

We fix $j$, and write $\tau=\tau_{j}, \gamma=\gamma_{j}$, etc. Since $N-1>p>1$, we have

$$
\int_{1}^{\infty}\left(1+t\left|\tau^{\prime}(t)\right|\right) t^{-N} d t \leqq \int_{1}^{\infty}\left(1+\left|\theta_{1}^{\prime}(t)\right|+\left|\theta_{2}^{\prime}(t)\right|\right) t^{1-N} d t<\infty
$$

by (i).

Notice that $\gamma((0, \infty)) \subset \Omega_{j}, \quad|\gamma(t)|=t, \quad$ and $\gamma^{\prime}(t)=\left\{1+i t \tau^{\prime}(t)\right\} e^{i \tau(t)}$ for all $t>0$ by (1). It follows from (b) that $s>r>C$ implies

$$
\begin{aligned}
|g(\gamma(s))-g(\gamma(r))| & =\left|\int_{r}^{s} g^{\prime}(\gamma(t)) \gamma^{\prime}(t) d t\right| \leqq \int_{r}^{s} C t^{-2 N}\left(1+t\left|\tau^{\prime}(t)\right|\right) d t \\
& \leqq C r^{-N} \int_{r}^{\infty}\left(1+t\left|\tau^{\prime}(t)\right|\right) t^{-N} d t .
\end{aligned}
$$

From (2) and (3) we infer that $g(\gamma(s))$ converges to some complex number $c=c_{j}$ as $s \rightarrow \infty$, and that

$$
|c-g(\gamma(r))|=o\left(r^{-N}\right) \quad \text { as } \quad r \rightarrow \infty .
$$

Now suppose that $z=r e^{i s} \in \Omega_{j}$, where $r>C$ and that $\theta_{j}(r)<s<\theta_{j+1}(r)$. Then we have

$$
|g(\gamma(r))-g(z)|=\left|\int_{s}^{\tau(r)} g^{\prime}\left(r e^{i t}\right) i r e^{i t} d t\right|<2 \pi C / r^{2 N-1}
$$

by (1) and (b), so

$$
|c-g(z)|<|c-g(\gamma(r))|+2 \pi C / r^{2 N-1} .
$$

This inequality, combined with (4), yields the desired conclusion.

Lemma 2. Let $\theta_{j}, p$, and $\Omega_{j}(j=1,2)$ be as in Lemma 1. Fix a positive real number $r_{0}$ and a natural number $N>p+1$. Let

$$
\alpha=r_{0} \exp \left[i \theta_{1}\left(r_{0}\right)\right] \text { and } \beta=r_{0} \exp \left[i \theta_{2}\left(r_{0}\right)\right] \text {, }
$$


and let $g(z)$ be a holomorphic antiderivative of $[(z-\alpha)(z-\beta)]^{-N}$ in the simply connected region

$$
\Omega_{0}=C \backslash \bigcup_{j=1}^{2}\left\{r \exp \left[i \theta_{j}(r)\right]: r \geqq r_{0}\right\} .
$$

Then there exist complex numbers $c_{1}, c_{2}$ such that

(c)

$$
g(z)=c_{j}+o\left(|z|^{-N}\right) \text { as } \quad z \in \Omega_{j} \quad \text { tends to } \infty
$$

for $j=1,2$. Moreover, $c_{1} \neq c_{2}$.

Proof. The existence of $c_{j}$ satisfying (c) is an immediate consequence of Lemma 1 . So we only need to check that $c_{1} \neq c_{2}$.

Let $\gamma_{1}$ and $\gamma_{2}$ be the two infinite curves defined as in the proof of Lemma 1. Notice that both $\gamma_{1}$ and $\gamma_{2}$ lie in $\Omega_{0}$, that $\gamma_{2}=-\gamma_{1}$ and that

$$
g(v)-g(u)=\int_{\Gamma}(z-\alpha)^{-N}(z-\beta)^{-N} d z \quad\left(u, v \in \Omega_{0}\right),
$$

where $\Gamma=\Gamma(u, v)$ is any smooth curve in $\Omega_{0}$ from $u$ to $v$. Now pick any $r>r_{0}$, and consider the closed curve $\gamma$ consisting of the following three pieces: $\gamma_{2}(r-t)$ for $0 \leqq t \leqq r, \gamma_{1}(t-r)$ for $r \leqq t \leqq 2 r$, and the semicircle

$$
C_{r}(t)=\gamma_{1}(r) \exp [i(t-2 r)] \text { for } 2 r \leqq t \leqq 2 r+\pi .
$$

It is easy to check that $\alpha$ and $\beta$ lie "outside" and "inside" of $\gamma$, respectively.

It follows from (1) and Cauchy's residue theorem that

where

$$
g\left(\gamma_{1}(r)\right)-g\left(\gamma_{2}(r)\right)+\int_{C_{r}}(z-\alpha)^{-N}(z-\beta)^{-N} d z=2 \pi i \operatorname{Res}(\beta),
$$

$$
\operatorname{Res}(\beta)=\frac{[2(N-1)] !}{[(N-1) !]^{2}}(-1)^{N-1}(\beta-\alpha)^{1-2 N} \neq 0 .
$$

But it is routine to show that $\lim r \rightarrow \infty \int_{C_{r}}=0$. Letting $r \rightarrow \infty$ in (2), we therefore conclude from (c) and (3) that $c_{1}-c_{2}+0 \neq 0$, as desired.

Now we write $P^{*}(w)=P(1 / w)$ for a polynomial $P$ and $w \neq 0$.

Lemma 3. Let $E$ be a closed subset of $C, K$ a compact connected subset of $C \backslash E$, and $u, v \in K$. If $N$ is a nonnegative integer, $\varepsilon>0$, and $R_{1}$ is a polynomial, then there exists a polynomial $R_{2}$ such that

$$
\left|R_{1}^{*}(z-u)-R_{2}^{*}(z-v)\right|<\varepsilon /(2+|z|)^{N} \quad \forall z \in E .
$$

Proof. For $N=0$, this is a consequence of Runge's theorem. (Indeed, it can be proved by an elementary method.) See, for example, Chapter IV, Paragraph 1 of S. Saks and A. Zygmund [9].

So assume that $N \geqq 1$ and that the result is true with $N$ replaced by $N-1$. Apply this inductive hypothesis to $R_{1}(z)=(u-v) z$ to find a polynomial $Q$ such that

$$
\left|(u-v)(z-u)^{-1}-Q^{*}(z-v)\right|<\varepsilon /(2+|z|)^{N-1} \quad \forall z \in E .
$$


Divide both sides of this inequality by $|z-v|$ to obtain

$$
\left|(z-u)^{-1}-\left\{(z-v)^{-1}+(z-v)^{-1} Q^{*}(z-v)\right\}\right|<\frac{\varepsilon}{|z-v|(2+|z|)^{N-1}} \quad \forall z \in E .
$$

But $(2+|z|) /|z-v|$ is bounded on $E$ and $\varepsilon>0$ is arbitrary. Thus we conclude that there exists a polynomial $R$ such that

$$
\left|(z-u)^{-1}-R^{*}(z-v)\right|<\varepsilon /(2+|z|)^{N} \quad \forall z \in E,
$$

which establishes the desired result for $R_{1}(z)=z$. Since $(z-u)^{-1}$ is bounded on $E$, (3) shows that $R^{*}(z-v)$ is bounded on $E$. Therefore the general case follows from this special case combined with the elementary formula $A^{n}-B^{n}=(A-B)\left(A^{n-1}+\ldots+\right.$ $\left.+B^{n-1}\right)$. This completes the induction and hence the proof.

Proof of the Theorem. First consider the case $k=1$. In this case we may assume that $\Omega_{1}$ is the complement of a curve $\Gamma$ of the form $\Gamma(t)=t e^{i \theta(t)}$ for $t \geqq 0$, where $\theta$ is in $S_{p}$ for some $p>0$. Then the hypothesis on the closed unbounded set $E_{1}$ is that it be disjoint from $\Gamma$. For such an $E_{1}$, it is easy to construct an infinitely differentiable function $\delta$ on $(0, \infty)$ such that $0<\delta(t)<2 \pi$ and $\left|\delta^{\prime}(t)\right|<1$ for all $t>0$ and such that

$$
E_{1} \subset\left\{r e^{i t}: r>0 \text { and } \theta(r)<t<\theta(r)+2 \pi-\delta(r)\right\} .
$$

Therefore the case $k=1$ can be reduced to the case $k=2$. Also the desired result for $k \geqq 3$ follows from $k$ applications of the result for $k=2$ as follows: for each pair of complementary spiral regions $\Omega_{j}$ and $C \backslash \bar{\Omega}_{j}$ and respective polynomials $P_{j}$ and 0 , the result for $k=2$ supplies us with an appropriate entire function $g_{j}$ and for the desired function $g$ we take $g_{1}+g_{2}+\ldots+g_{k}$. Thus it will be sufficient to deal with the case $k=2$.

So assume that $k=2$ and also, without loss of generality, that $\Omega_{1}$ and $\Omega_{2}$ are defined by (a) in Lemma 1 . Let $E_{j}$ be a closed unbounded set contained in $\Omega_{j}$ for $j=1,2$. Choose and fix an infinitely differentiable function $\delta$ on $(0, \infty)$, with bounded derivative, such that

$$
\begin{gathered}
0<\delta<4^{-1} \min \left\{\theta_{2}-\theta_{1}, \theta_{3}-\theta_{2}\right\} \text { on }(0, \infty) \text {, and for } j=1,2 \\
E_{j} \subset\left\{r e^{i t}: r>0 \text { and } \theta_{j}^{+}(r)<t<\theta_{j+1}^{-}(r)\right\} \stackrel{\text { def }}{=} \Omega_{j}^{*},
\end{gathered}
$$

where

$$
\theta_{j}^{+}=\theta_{j}+\delta \quad \text { and } \quad \theta_{j}^{-}=\theta_{j}-\delta \quad(j=1,2,3) .
$$

Now let $\varepsilon>0$ and a natural number $q>p+1$ be given. Put

$$
\begin{gathered}
U_{n}=\left\{r e^{i t}: r>n-1 / 2,\left|t-\theta_{1}(t)\right|<\delta(r)\right\}, \\
V_{n}=\left\{r e^{i t}: r>n-1 / 2,\left|t-\theta_{2}(r)\right|<\delta(r)\right\}, \\
\alpha_{n}=n e^{i \theta_{1}(n)} \text { and } \beta_{n}=n e^{i \theta_{2}(n)} \text { for } n=1,2, \ldots .
\end{gathered}
$$


We shall construct two sequences of polynomials $Q_{n}$ and $R_{n}$ as follows. The rational function $f_{1}(z)=\left[\left(z-\alpha_{1}\right)\left(z-\beta_{1}\right)\right]^{-q}$ admits a representation of the form

$$
f_{1}(z)=Q_{1}^{*}\left(z-\alpha_{1}\right)+R_{1}^{*}\left(z-\beta_{1}\right)
$$

for some polynomials $Q_{1}$ and $R_{1}$. Suppose that polynomials $Q_{n}$ and $R_{n}$ have been chosen for some $n \geqq 1$. Notice that $\alpha_{n}$ and $\alpha_{n+1}$ are contained in an arc which is disjoint from the closed set $C \backslash U_{n}$. It follows from Lemma 3 that there exists a polynomial $Q_{n+1}$ such that

$$
\left|Q_{n+1}^{*}\left(z-\alpha_{n+1}\right)-Q_{n}^{*}\left(z-\alpha_{n}\right)\right|<\varepsilon /(2+|z|)^{2 q n} \quad \forall z \in C \backslash U_{n} .
$$

Similarly there exists a polynomial $R_{n+1}$ such that

$$
\left|R_{n+1}^{*}\left(z-\beta_{n+1}\right)-R_{n}^{*}\left(z-\beta_{n}\right)\right|<\varepsilon /(2+|z|)^{2 q n} \quad \forall z \in C \backslash V_{n} .
$$

This completes our induction.

Now set $f_{n}(z)=Q_{n}^{*}\left(z-\alpha_{n}\right)+R_{n}^{*}\left(z-\beta_{n}\right)$ for $n=1,2, \ldots$ Then (8) and (9) yield

$$
\left|f_{n+1}(z)-f_{n}(z)\right|<2 \varepsilon /(2+|z|)^{2 q n} \quad \forall z \in C \backslash\left(U_{n} \cup V_{n}\right) .
$$

It follows from (4), (5) and (10) that the rational functions $f_{n}$ converge to an entire function $f$ uniformly on each compact set. Moreover, we have

$$
\left|f(z)-f_{1}(z)\right|<\sum_{n=1}^{\infty} \frac{2 \varepsilon}{(2+|z|)^{2 q n}} \leqq \frac{4 \varepsilon}{(2+|z|)^{2 q}} \quad \forall z \in C \backslash\left(U_{1} \cup V_{1}\right) .
$$

Let $g$ be the antiderivative of $f$ with $g(0)=0$, and let $g_{1}$ be the antiderivative of $f_{1}$ with $g_{1}(0)=0$ in

$$
C \backslash \bigcup_{j=1}^{2}\left\{t \exp \left[i \theta_{j}(t)\right]: t \geqq 1\right\}
$$

By Lemma 2, there exist distinct complex numbers $c_{1}$ and $c_{2}$ such that

$$
g_{1}(z)=c_{j}+o\left(|z|^{-q}\right) \quad \text { as } \quad z \in \Omega_{j} \text { tends to } \infty
$$

for $j=1,2$. By the definition of $f_{1}$ and (11), we can find $C>1$ so large that $|f(z)|<$ $C /|z|^{2 q}$ for all $z$ in $C \backslash\left(U_{1} \cup V_{1}\right)$ with $|z|>C$. It follows from (two applications of) Lemma 1 and (1)-(5) that there exist two complex numbers $b_{1}$ and $b_{2}$ such that

$$
g(z)=b_{j}+o\left(|z|^{-q}\right) \text { as } z \in \Omega_{j}^{*} \text { tends to } \infty
$$

for $j=1,2$. We claim that $b_{1} \neq b_{2}$, provided that $\varepsilon>0$ is small enough.

Indeed, let $\tau_{j}$ and $\gamma_{j}$ be as in the proof of Lemma 1:

$$
\tau_{j}(t)=\left\{\theta_{j}(t)+\theta_{j+1}(t)\right\} / 2 \text { and } \gamma_{j}(t)=t e^{i \tau_{j}(t)} \text { for } t \geqq 0 .
$$

According to (1)-(5) we have

$$
\gamma_{j}(0, \infty) \subset \Omega_{j}^{*} \subset C \backslash\left(U_{1} \cup V_{1}\right), \quad j=1,2 .
$$


Since $g(0)=g_{1}(0)=0$, it follows from (11) and (14) that $r>0$ implies

$$
\begin{gathered}
\left|g\left(\gamma_{j}(r)\right)-g_{1}\left(\gamma_{j}(r)\right)\right|=\left|\int_{0}^{r}\left\{f\left(\gamma_{j}(t)\right)-f_{1}\left(\gamma_{j}(t)\right)\right\} \gamma_{j}^{\prime}(t) d t\right| \\
\quad<4 \varepsilon \int_{0}^{\infty} \frac{1+t\left(\left|\theta_{1}^{\prime}(t)\right|+\left|\theta_{2}^{\prime}(t)\right|\right)}{(2+|t|)^{2 q}} d t=4 \varepsilon B, \quad \text { say. }
\end{gathered}
$$

Notice that $B$ is a finite constant which is independent of $\varepsilon$. Letting $r \rightarrow \infty$ in (15) we obtain from (12) and (13) that $\left|b_{j}-c_{j}\right| \leqq 4 \varepsilon B$ for $j=1,2$. Hence $\left|b_{1}-b_{2}\right| \geqq$ $\left|c_{1}-c_{2}\right|-8 \varepsilon B>0$, provided that $\varepsilon>0$ is small enough, which confirms our claim. Upon setting $h=\alpha+\beta g$ for appropriate coefficients $\alpha$ and $\beta$, we therefore obtain an entire function $h$ such that

$$
h(z)=\left\{\begin{array}{llll}
a+o\left(|z|^{-q}\right) & \text { as } & z \in E_{1} & \text { tends to } \infty, \text { and } \\
b+o\left(|z|^{-q}\right) & \text { as } & z \in E_{2} & \text { tends to } \infty,
\end{array}\right.
$$

where $a$ and $b$ are arbitrary, but preassigned, complex numbers.

Finally let $P_{1}$ and $P_{2}$ be two given polynomials. Write

$$
P_{1}(z)=\sum_{k=0}^{M} a_{k} z^{k} \quad \text { and } \quad P_{2}(z)=\sum_{k=0}^{M} b_{k} z^{k} .
$$

Choose a natural number $q>M+N+p$ and entire functions $h_{k}$ which behave as in (16) with $a=a_{k}$ and $b=b_{k}(k=0,1, \ldots, M)$. Put $F(z)=\sum_{0}^{M} z^{k} h_{k}(z)$. It is evident that $F$ has the required properties.

Proof of the Corollary. Let $f$ be any continuously differentiable, positive realvalued function on $(0, \infty)$ for which there exists $p>0$ such that

$$
x+f^{\prime}(x) f(x)>0 \text { for all } x \geqq p,
$$

and

$$
\int_{p}^{\infty}\left|f^{\prime}(x) x-f(x)\right| \cdot\left(x^{2}+f(x)^{2}\right)^{-p / 2-2} d x<\infty .
$$

The graph of such a function is essentially a curve of the kind which bounds spiral regions. To see this, define two functions $t$ and $\theta$ of $x$ by setting $t(x)=\left(x^{2}+f(x)^{2}\right)^{1 / 2}$ and $\theta(x)=\arctan [f(x) / x]$. Then $d t / d x=\left[x+f^{\prime}(x) f(x)\right] / t>0$ for all $x \geqq p$ by (1), so $t$ has a continuously differentiable inverse on $[b, \infty)$, where $b=t(p)$. Accordingly, $\theta$ may be regarded as a function of $t \geqq b$. A simple calculation shows that

$$
\begin{aligned}
& \int_{b}^{\infty}\left|\frac{d \theta}{d t}\right| t^{-p} d t=\int_{p}^{\infty}\left|\frac{d \theta}{d x}\right| t^{-p} d x \\
= & \int_{p}^{\infty}\left|f^{\prime}(x) x-f(x)\right|\left(x^{2}+f(x)^{2}\right)^{-p / 2-2} d x,
\end{aligned}
$$

which is finite by (2). Thus (after an extension to $(0, b)$ ) the function $\theta$ belongs to $S_{p}$.

It is evident that $e^{x}$ satisfies (1) and (2) for all $p>0$. Put

$$
H=\left\{(x, y): x \geqq 1 \text { and } 2^{-1} e^{x} \leqq y \leqq 2 e^{x}\right\} .
$$

By the result established in the preceding paragraph, $C \backslash H$ is essentially contained in a spiral region $\Omega$. (More precisely, $C \backslash H$ is contained in the union of $\Omega$ and a 
bounded set.) Given a natural number $N$, our theorem yields an entire function $g$ such that $g(z)=o\left(|z|^{-N}\right)$ as $z \in \Omega$ tends to $\infty$. Indeed, such a $g$ can be chosen so that $g(z)=1+o\left(|z|^{-N}\right)$ as $z \rightarrow \infty$ along the curve $y=e^{x}$ for $x>0$.

Now let $\Gamma$ be an arbitrary algebraic curve. Thus, by definition, there exist finitely many polynomials $Q_{0}, Q_{1}, \ldots, Q_{n}$ in $x$, with $Q_{n} \neq 0$, such that each point $(x, y)$ of $\Gamma$ satisfies

$$
Q_{0}(x)+Q_{1}(x) y+\ldots+Q_{n}(x) y^{n}=0 .
$$

It is easily seen from (3) that if $(x, y)$ is in $H$ and $x$ or $y$ is large enough, then $(x, y)$ does not satisfy (4). In other words, only a bounded portion of $\Gamma$ lies outside $\Omega$; hence $g(z)=o\left(|z|^{-N}\right)$ as $z \rightarrow \infty$ along $\Gamma$. It follows that, given a polynomial $P$, the entire function $g+P$ has the required properties.

\section{References}

[1] Bieberbach, L. : Neuere Untersuchungen über Funktionen von komplexen Variablen. - Encyklopädie der Mathematischen Wissenschaften II C 4, B. G. Teubner, Leipzig, 1921, $379-532$.

[2] BoHR, H.: Über ganze transzendente Funktionen von einem besonderen Typus. Beispiel einer allgemeinen Konstruktionsmethode. S.-B. Preuss. Akad. Wiss. 26, 1929, 565-571.

[3] BoHr, H.: Collected mathematical works, vol. III. - Dansk Matematisk Forening, København, 1952.

[4] Bouligand, G.: Sur certaines fonctions entières. - Bull. Math. Fac. Sci. Grandes Écoles 1, $1934,65-70$.

[5] Grandjot, K.: Über Grenzwerte ganzer transzendenter Funktionen. - Math. Ann. 91, 1924, $316-320$.

[6] Newman, D. J.: An entire function bounded in every direction. - Amer. Math. Monthly 83, 1976, 192-193.

[7] Pólya, G.: Fonctions entières (question 2917, de M. Petrovitch). - Intermédiaire des mathématiciens (2) $1,1922,81-82$.

[8] Pólya, G., and G. Szegő,: Problems and theorems of analysis vol. II. - Die Grundlehren der mathematischen Wissenschaften 216, Springer-Verlag, Berlin-Heidelberg-New York, 1976.

[9] SAKs, S., and A. Zygmund: Analytic functions, 3rd ed. - Elsevier Publishing Co., AmsterdamLondon-New York, 1971.

[10] SCHNeIDER, W. J.: Approximation and harmonic measure. - Aspects of Contemporary Complex Analysis, Proceedings of an Instructional Conference organized by the London Mathematical Society at the University of Durham, edited by D. A. Brannan and J. G. Clunie, Academic Press, London-New York-Toronto-Sydney-San Francisco, 1980, 333-349.

Kansas State University

Department of Mathematics

Manhattan, Kansas 66506

USA

Received 31 January 1984 